\title{
Survival with Cardiac-Resynchronization Therapy in Mild Heart Failure
}

\author{
Ilan Goldenberg, M.D., Valentina Kutyifa, M.D., Ph.D., Helmut U. Klein, M.D., \\ David S. Cannom, M.D., Mary W. Brown, M.S., Ariela Dan, Ph.D., \\ James P. Daubert, M.D., N.A. Mark Estes III, M.D., Elyse Foster, M.D., \\ Henry Greenberg, M.D., Josef Kautzner, M.D., Robert Klempfner, M.D., \\ Malte Kuniss, M.D., Bela Merkely, M.D., Ph.D., Marc A. Pfeffer, M.D., Ph.D., \\ Aurelio Quesada, M.D., Ph.D., Sami Viskin, M.D., Scott McNitt, M.S., \\ Bronislava Polonsky, M.S., Ali Ghanem, M.D., Scott D. Solomon, M.D., \\ David Wilber, M.D., Wojciech Zareba, M.D., Ph.D., and Arthur J. Moss, M.D.
}

A B STRACT

The authors' affiliations are listed in the Appendix. Address reprint requests to Dr. Goldenberg at Leviev Heart Center and Israeli Association for Cardiovascular Trials, Sheba Medical Center and Tel Aviv University, Tel Hashomer 5265601, Israel, or at ilan.goldenberg@sheba.health.gov.il; or to Dr. Moss at the Heart Research Follow-up Program, University of Rochester Medical Center, 265 Crittenden Blvd., CU 420653, Rochester, NY 14642-0653, or at heartajm@heart.rochester.edu.

Drs. Goldenberg, Kutyifa, Klein, and Moss contributed equally to this article.

This article was published on March 30, 2014, at NEJM.org.

N EngIJ Med 2014;370:1694-701. DOI: 10.1056/NEJMoal401426

Copyright (c) 2014 Massachusetts Medical Society.

\section{BACKGROUND}

The Multicenter Automatic Defibrillator Implantation Trial with Cardiac Resynchronization Therapy (MADIT-CRT) showed that early intervention with cardiac-resynchronization therapy with a defibrillator (CRT-D) in patients with an electrocardiographic pattern showing left bundle-branch block was associated with a significant reduction in heart-failure events over a median follow-up of 2.4 years, as compared with defibrillator therapy alone.

\section{METHODS}

We evaluated the effect of CRT-D on long-term survival in the MADIT-CRT population. Post-trial follow-up over a median period of 5.6 years was assessed among all 1691 surviving patients (phase 1) and subsequently among 854 patients who were enrolled in post-trial registries (phase 2). All reported analyses were performed on an intention-to-treat basis.

\section{RESULTS}

At 7 years of follow-up after initial enrollment, the cumulative rate of death from any cause among patients with left bundle-branch block was $18 \%$ among patients randomly assigned to CRT-D, as compared with $29 \%$ among those randomly assigned to defibrillator therapy alone (adjusted hazard ratio in the CRT-D group, 0.59; 95\% confidence interval [CI], 0.43 to $0.80 ; \mathrm{P}<0.001$ ). The long-term survival benefit of CRT-D in patients with left bundle-branch block did not differ significantly according to sex, cause of cardiomyopathy, or QRS duration. In contrast, CRT-D was not associated with any clinical benefit and possibly with harm in patients without left bundlebranch block (adjusted hazard ratio for death from any cause, 1.57; 95\% CI, 1.03 to 2.39; $\mathrm{P}=0.04 ; \mathrm{P}<0.001$ for interaction of treatment with $\mathrm{QRS}$ morphologic findings).

CONCLUSIONS

Our findings indicate that in patients with mild heart-failure symptoms, left ventricular dysfunction, and left bundle-branch block, early intervention with CRT-D was associated with a significant long-term survival benefit. (Funded by Boston Scientific; ClinicalTrials.gov numbers, NCT00180271, NCT01294449, and NCT02060110.) 
I HE MULTICENTER AUTOMATIC DEFIbrillator Implantation Trial with Cardiac Resynchronization Therapy (MADIT-CRT) showed the safety and effectiveness of cardiacresynchronization therapy (CRT) with a defibrillator (CRT-D) in patients with asymptomatic or mildly symptomatic heart failure, a reduced ejection fraction, and a prolonged QRS duration. ${ }^{1}$ The study showed that treatment with CRT-D was associated with a $34 \%$ relative reduction in the risk of nonfatal heart-failure events or death from any cause, as compared with implantable cardioverterdefibrillator (ICD) therapy alone over a median follow-up period of 2.4 years. The benefit of CRT-D in the trial was primarily driven by a significant relative reduction of $41 \%$ in the risk of nonfatal heart-failure events ${ }^{1}$ and was subsequently shown to be restricted to patients with an electrocardiographic (ECG) pattern showing left bundle-branch block. ${ }^{2}$ Thus, questions regarding the ability of CRT to reduce mortality among patients with asymptomatic or mildly symptomatic heart failure remained unanswered. In the present study, we prospectively assessed the long-term outcome of the patients enrolled in MADIT-CRT.

\section{METHODS}

\section{STUDY POPULATION}

The design, protocol, and results of MADIT-CRT have been published previously. ${ }^{1,3}$ Briefly, 1820 patients with ischemic cardiomyopathy (New York Heart Association [NYHA] functional class I or II) or nonischemic cardiomyopathy (NHYA functional class II only), a left ventricular ejection fraction of $30 \%$ or less, and a prolonged QRS duration $(\geq 130 \mathrm{msec}$ ) were randomly assigned in a 3:2 ratio to receive CRT-D or ICD therapy. All eligible patients met the guideline criteria for ICD therapy. Of the 1820 patients who were enrolled in MADIT-CRT, 1271 (70\%) were enrolled at 88 centers in the United States, and 549 (30\%) were enrolled at 24 centers in Europe, Israel, and Canada.

\section{DATA ACQUISITION AND PATIENT FOLLOW-UP}

MADIT-CRT was carried out from December 22, 2004, through June 22, 2009. After the publication of the primary results, ${ }^{1}$ post-trial follow-up was conducted for all 1691 surviving study participants until September 10, 2010 (phase 1 of the extended follow-up). After September 10, 2010, ongoing patient follow-up (phase 2) was conducted at the 48 U.S. centers that agreed to participate in the long-term follow-up requested by the Food and Drug Administration for patients enrolled in the United States (coordinated by the Heart Research Follow-up Program at the University of Rochester Medical Center, Rochester, New York) and at 23 of the 24 non-U.S. centers (coordinated by the Israeli Association for Cardiovascular Trials at Sheba Medical Center, Tel Hashomer, Israel), involving a total of 854 patients. A total of 40 U.S. centers declined to participate in the phase 2 registry.

The clinical characteristics of the patients who enrolled and those who did not enroll in phase 2 of the post-trial follow-up are shown in Table S1 in the Supplementary Appendix, available with the full text of this article at NEJM.org. Both phases of the post-trial follow-up were approved by the institutional review board at each participating center, and all the patients provided written informed consent. The numbers of patients enrolled in the trial and in the post-trial followup phases are shown in Figure 1.

All the authors were involved in the study design, data collection, and analysis; participated in the writing of the manuscript; and made the decision to submit the manuscript for publication. The commercial sponsor had no role in the study design, data accrual or analysis, or manuscript preparation or review. All the authors vouch for the accuracy and completeness of the reported findings and for the fidelity of the study to the protocol.

\section{DEFINITIONS AND END POINTS}

The original MADIT-CRT protocol did not specify the evaluation of a potential differential effect of CRT-D with respect to QRS morphologic findings at baseline (in other respects, the current report is true to the protocol). However, after the publication of the primary report, ${ }^{1}$ analyses were performed comparing patients who had left bundle-branch block ( $70 \%$ of the study patients) with those who did not have left bundle-branch block (30\%, including $13 \%$ with right bundle-branch block and $17 \%$ with an intraventricular conduction delay), which suggested that the reduced risk of the primary end point with CRT-D was largely, or even entirely, in the subgroup of patients with left bundle-branch block. ${ }^{2}$

On the basis of this post-trial observation, the analysis in the present study evaluated the dif- 


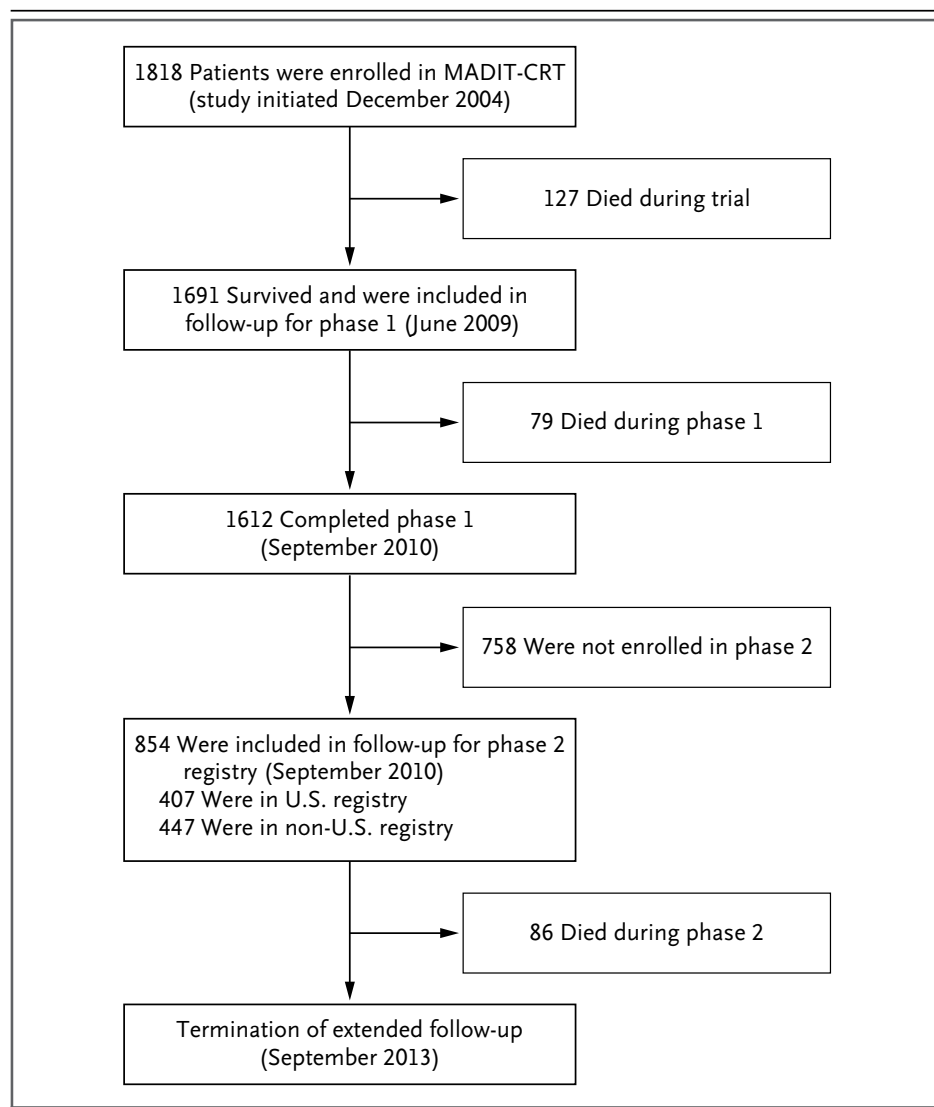

Figure 1. Study Design.

The population of the present study consisted of all the patients included in the Multicenter Automatic Defibrillator Implantation Trial with Cardiac Resynchronization Therapy (MADIT-CRT), ${ }^{1}$ with the exclusion of two patients for whom baseline electrocardiographic data regarding QRS morphologic findings were not available. ferential effects of CRT-D according to baseline QRS morphologic findings - specifically, left bundle-branch block or no left bundle-branch block. Two patients with missing data regarding QRS pattern at enrollment were excluded from the present study. Thus, the final study sample included the 1818 patients from MADIT-CRT for whom baseline ECG data were available.

The primary end point of the current study was death from any cause. Secondary end points included a nonfatal heart-failure event and the combined end point of a nonfatal heart-failure event or death, whichever occurred first.

\section{STATISTICAL ANALYSIS}

All analyses in the present study were carried out on an intention-to-treat basis (i.e., according to the original treatment assignment, regardless of in- trial or post-trial crossovers). Variables were expressed as means $\pm S D$, and categorical data were summarized as frequencies and percentages. The clinical characteristics of the patients at baseline were compared between the subgroups, with the use of the Kruskal-Wallis test for continuous variables and the chi-square test or Fisher's exact test for dichotomous variables.

The Kaplan-Meier method was used to determine cumulative probabilities of death from any cause and nonfatal heart-failure events from the time of enrollment in MADIT-CRT through posttrial follow-up, according to treatment group, with between-group comparisons of cumulative event rates calculated by means of the log-rank test. The number of patients who would need to be treated with CRT-D to save one life was calculated as the inverse of the survival difference between the two treatment groups at each time point.

Multivariate Cox proportional-hazards regression analyses were used to evaluate the effect of CRT-D on the two end points of death from any cause and a nonfatal heart-failure event and on the combined end point of a nonfatal heart-failure event or death (whichever came first), from the time of enrollment in MADIT-CRT through posttrial follow-up. The Cox model was adjusted for relevant clinical covariates with the use of bestsubset regression modeling (including age, serum creatinine level, presence or absence of diabetes mellitus, cause of cardiomyopathy, left ventricular end-systolic volume, QRS duration, NYHA functional class 3 months before enrollment, and smoking status). The benefit of CRT-D therapy as compared with ICD therapy alone among patients with left bundle-branch block and those without left bundle-branch block was assessed by including a term for interaction between treatment and presence or absence of left bundle-branch block in the multivariate Cox models.

Since the assessment of the interaction between QRS morphologic findings and treatment was not prespecified in the original protocol, we also carried out a post-trial landmark analysis (i.e., with the follow-up time beginning on June 23, 2009, which was after trial closure on June 22, 2009). The landmark Cox model was adjusted for the same covariates listed above and for the term for interaction between treatment and presence or absence of left bundle-branch block.

All statistical tests were two-sided, and a P value of less than 0.05 was considered to indicate 
statistical significance. The P values for interaction are reported. Analyses were carried out with the use of SAS software, version 9.3 (SAS Institute).

RESULTS

\section{PATIENTS}

Phase 1 of the post-trial follow-up was carried out from June 2009 through September 2010 and included all surviving patients enrolled in MADIT-CRT; phase 2 was conducted through September 2013. The median follow-up of the enrolled patients during the trial was 2.4 years (interquartile range, 1.8 to 3.2 ), and the median follow-up after the trial was 5.6 years (interquartile range, 5.1 to 6.4 ). The clinical characteristics of the 854 patients who participated in phase 2 of the extended follow-up were similar in the ICDonly and CRT-D groups (Table 1).

During long-term follow-up, 171 of the 1818 patients (9\%) crossed over from ICD therapy alone to CRT-D therapy, and 92 (5\%) crossed over from CRT-D to ICD therapy alone. Patients who crossed over from ICD to CRT-D therapy had several baseline characteristics that put them at higher risk than those who crossed over from CRT-D to ICD or those who did not cross over, including a higher serum creatinine level, a lower ejection fraction, and larger left ventricular volumes.

\section{CRT-D IN PATIENTS WITH LEFT BUNDLE-BRANCH BLOCK}

Kaplan-Meier estimates of death from any cause during the period from enrollment through 7 years of follow-up in the two treatment groups are shown in Figure 2A. The curves diverge at 1 year and continue to have separate paths thereafter, with significantly lower mortality among patients randomly assigned to CRT-D therapy than among those randomly assigned to ICD therapy alone $(\mathrm{P}=0.002$ by the log-rank test without adjustment). Thus, at 7 years of follow-up, the cumulative rate of death from any cause among patients with left bundle-branch block was $29 \%$ in the ICD-only group, as compared with $18 \%$ in the CRT-D group. The survival difference corresponded to nine patients who would need to be treated with CRT-D to save one life within 7 years.

The cumulative probability of a nonfatal heartfailure event during 7 years of follow-up was also significantly lower among patients randomly as-
Table 1. Clinical, Electrocardiographic, and Echocardiographic Characteristics of the Patients Enrolled in Phase 2 of the Extended Follow-up.*

\begin{tabular}{|c|c|c|c|}
\hline Characteristic & $\begin{array}{l}\text { ICD-Only } \\
\text { Group } \\
(\mathrm{N}=327)\end{array}$ & $\begin{array}{c}\text { CRT-D } \\
\text { Group } \\
(\mathrm{N}=527)\end{array}$ & $P$ Value \\
\hline & \multicolumn{2}{|c|}{ no. (\%) } & \\
\hline Female sex & $75(23)$ & $128(24)$ & 0.65 \\
\hline Age $\geq 65 \mathrm{yr}$ & $158(48)$ & $262(50)$ & 0.69 \\
\hline Ischemic cardiomyopathy & $166(51)$ & $279(53)$ & 0.54 \\
\hline NYHA class I & $51(16)$ & $73(14)$ & 0.48 \\
\hline $\begin{array}{l}\text { NYHA class III or IV }>3 \text { mo before } \\
\text { enrollment }\end{array}$ & $39(12)$ & $53(10)$ & 0.38 \\
\hline QRS duration $<150 \mathrm{msec}$ & $103(31)$ & $164(31)$ & 0.91 \\
\hline Left ventricular ejection fraction $\leq 25 \%$ & $210(64)$ & $324(61)$ & 0.42 \\
\hline Left bundle-branch block & $240(73)$ & $394(75)$ & 0.66 \\
\hline Heart rate $\geq 80$ beats $/ \mathrm{min}$ & $46(14)$ & $73(14)$ & 0.91 \\
\hline Body-mass index $\geq 30 \uparrow$ & $109(33)$ & $178(34)$ & 0.83 \\
\hline Blood urea nitrogen $>25 \mathrm{mg} / \mathrm{dl} \psi$ & $77(24)$ & $125(24)$ & 0.99 \\
\hline Serum creatinine $\geq 1.4 \mathrm{mg} / \mathrm{dl} \mathbb{S}$ & $60(18)$ & $90(17)$ & 0.64 \\
\hline Previous CABG & $76(23)$ & $145(28)$ & 0.17 \\
\hline Diabetes mellitus & $77(24)$ & $152(29)$ & 0.10 \\
\hline Hypertension & $207(63)$ & $340(65)$ & 0.74 \\
\hline Previous myocardial infarction & $129(40)$ & $223(43)$ & 0.47 \\
\hline Current smoker & $39(12)$ & $56(11)$ & 0.53 \\
\hline \multicolumn{4}{|l|}{ Previous arrhythmia } \\
\hline Atrial & $39(12)$ & $54(10)$ & 0.45 \\
\hline Ventricular & $23(7)$ & $44(8)$ & 0.50 \\
\hline \multicolumn{4}{|l|}{ Medications } \\
\hline ACE inhibitor & $250(76)$ & $420(80)$ & 0.26 \\
\hline ARB & $73(22)$ & $104(20)$ & 0.36 \\
\hline ACE inhibitor or ARB & $318(97)$ & $510(97)$ & 0.70 \\
\hline Amiodarone & $29(9)$ & $34(6)$ & 0.19 \\
\hline Beta-blocker & $311(95)$ & $498(94)$ & 0.70 \\
\hline Diuretic & $233(71)$ & 359 (68) & 0.33 \\
\hline
\end{tabular}

* ACE denotes angiotensin-converting enzyme, ARB angiotensin-receptor blocker, CABG coronary-artery bypass grafting, CRT-D cardiac-resynchronization therapy with defibrillator, ICD implantable cardioverter-defibrillator, and NYHA New York Heart Association.

$\uparrow$ The body-mass index is the weight in kilograms divided by the square of the height in meters.

$¥$ To convert the value for blood urea nitrogen to millimoles per liter, multiply by 0.357 .

$\int$ To convert the value for creatinine to millimoles per liter, multiply by 88.4

9 Data were missing for 16 patients (8 patients in each group).

signed to CRT-D than among those randomly assigned to ICD therapy alone $(\mathrm{P}<0.001$ by the log-rank test without adjustment, for the overall difference during follow-up). The event rates sepa- 


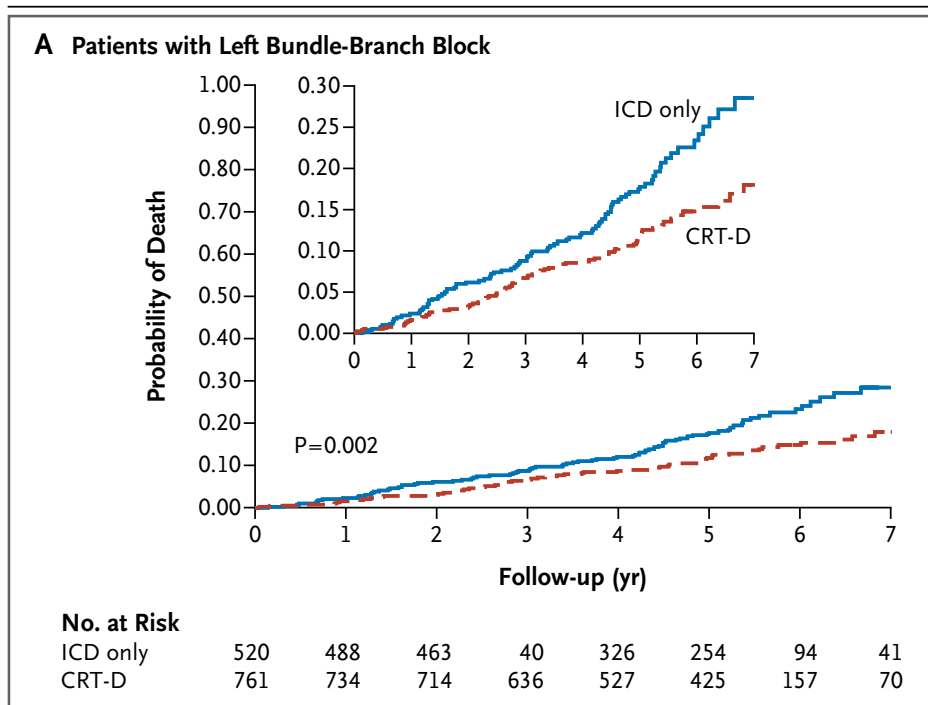

B Patients without Left Bundle-Branch Block

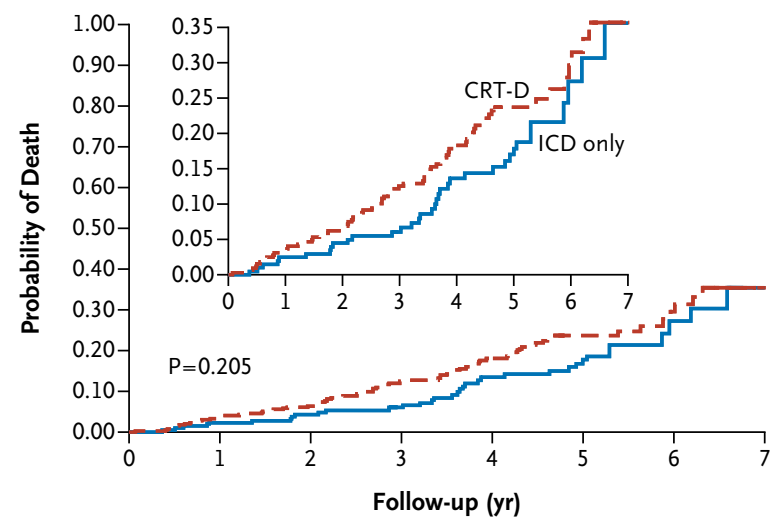

No. at Risk

ICD only

CRT-D

209

328

197

189

292

156

115

18

95

Figure 2. Kaplan-Meier Estimates of the Cumulative Probability of Death from Any Cause among Patients with and Those without Left BundleBranch Block.

CRT-D denotes cardiac-resynchronization therapy with defibrillator, and ICD implantable cardioverter-defibrillator. The insets show the same data on an enlarged y axis. signed to CRT-D therapy, as compared with those randomly assigned to ICD therapy alone. For the secondary end point of a nonfatal heart-failure event, the adjusted hazard ratio of 0.38 indicated a reduction in risk of $62 \%$ with CRT-D (Table 2).

The effects of CRT-D therapy on mortality among patients with left bundle-branch block in seven prespecified subgroups are shown in Figure 3. The survival benefit with CRT-D was consistent in each subgroup analyzed, including patients with ischemic cardiomyopathy and those with nonischemic cardiomyopathy, men and women, and patients with a longer QRS duration ( $\geq 150 \mathrm{msec}$ ) and those with a shorter QRS duration $(<150 \mathrm{msec})$; there were no significant treatment-by-subgroup interactions. The survival benefit provided by CRT-D in patients with left bundle-branch block was independent of the QRS duration, even when the QRS duration was further categorized into quartiles (Fig. S2 in the Supplementary Appendix).

\section{CRT-D IN PATIENTS WITHOUT LEFT BUNDLE-BRANCH} BLOCK

Among patients without left bundle-branch block, Kaplan-Meier survival analysis did not show a significant difference between the two treatment groups in the cumulative probability of death from any cause during the period from enrollment through 7 years of follow-up $(\mathrm{P}=0.21$ by the logrank test without adjustment) (Fig. 2B). Similarly, the cumulative probability of a nonfatal heartfailure event during 7 years of follow-up did not differ significantly between the ICD-only group and the CRT-D group $(\mathrm{P}=0.58$ by the log-rank test without adjustment) (Fig. S3 in the Supplementary Appendix).

Cox proportional-hazards regression modeling consistently showed a lack of benefit associated with CRT-D in patients without left bundlebranch block, with a trend toward an increased risk of death observed only after multivariate adjustment (Table 2). The differential effect of CRT-D on outcomes according to QRS morphologic findings was significant for all end points $(\mathrm{P}<0.05$ for interaction of treatment with $\mathrm{QRS}$ morphologic findings, in unadjusted and adjusted analyses) (Table 2). The lack of a survival benefit associated with CRT-D in patients without left bundle-branch block was consistent among those with a longer QRS duration ( $\geq 150 \mathrm{msec}$ ) or a shorter QRS duration ( $<150 \mathrm{msec}$ ), and among patients with QRS morphologic findings show- 


\begin{tabular}{|c|c|c|c|c|c|c|c|}
\hline \multirow[t]{2}{*}{ End Point } & \multirow[t]{2}{*}{$\begin{array}{l}\text { No. of } \\
\text { Events }\end{array}$} & \multirow[t]{2}{*}{$\begin{array}{l}\text { No. of } \\
\text { Patients }\end{array}$} & \multicolumn{2}{|c|}{ Left Bundle-Branch Block } & \multicolumn{2}{|c|}{ Non-Left Bundle-Branch Block } & \multirow[t]{2}{*}{$\begin{array}{l}\text { P Value for } \\
\text { Interaction* }\end{array}$} \\
\hline & & & $\begin{array}{l}\text { Hazard Ratio } \\
(95 \% \mathrm{Cl})\end{array}$ & P Value & $\begin{array}{l}\text { Hazard Ratio } \\
\quad(95 \% \mathrm{Cl})\end{array}$ & P Value & \\
\hline \multicolumn{8}{|l|}{ Death from any cause } \\
\hline Unadjusted analysis & 292 & 1818 & $0.63(0.47-0.84)$ & 0.002 & $1.31(0.87-1.96)$ & 0.19 & 0.004 \\
\hline Adjusted analysis $\uparrow$ & 267 & 1681 & $0.59(0.43-0.80)$ & $<0.001$ & 1.57 (1.03-2.39) & 0.04 & $<0.001$ \\
\hline \multicolumn{8}{|c|}{ Nonfatal heart-failure event } \\
\hline Unadjusted analysis & 442 & 1818 & $0.42(0.33-0.52)$ & $<0.001$ & $1.10(0.79-1.53)$ & 0.59 & $<0.001$ \\
\hline Adjusted analysis ${ }^{\dagger}$ & 405 & 1681 & $0.38(0.30-0.48)$ & $<0.001$ & $1.13(0.80-1.60)$ & 0.48 & $<0.001$ \\
\hline \multicolumn{8}{|l|}{$\begin{array}{l}\text { Nonfatal heart-failure event } \\
\text { or death }\end{array}$} \\
\hline Unadjusted analysis & 577 & 1818 & $0.50(0.41-0.61)$ & $<0.001$ & $1.21(0.90-1.63)$ & 0.21 & $<0.001$ \\
\hline Adjusted analysis $†$ & 530 & 1681 & $0.45(0.37-0.56)$ & $<0.001$ & $1.27(0.94-1.73)$ & 0.12 & $<0.001$ \\
\hline
\end{tabular}

* The $P$ value for interaction represents the likelihood that the difference between the treatment effect on patients with left bundle-branch block and the treatment effect on those without left bundle-branch block occurred by chance alone.

$\dagger$ Models were adjusted for the following covariates: age at enrollment, serum creatinine level of $1.4 \mathrm{mg}$ per deciliter (120 $\mu \mathrm{mol}$ per liter) or more, smoking status at enrollment, presence or absence of diabetes mellitus, cause of cardiomyopathy (ischemic vs. nonischemic), left ventricular endsystolic volume at baseline indexed by body-surface area, QRS duration at baseline ( $\geq 150 \mathrm{msec} v \mathrm{~s} .<150 \mathrm{msec}$ ), and NYHA class III or IV more than 3 months before enrollment (yes vs. no). Covariates were identified from a best-subset regression analysis.

ing right bundle-branch block or intraventricular conduction delay (Fig. S4 in the Supplementary Appendix).

\section{POST-TRIAL ANALYSIS}

To further validate the consistency of our findings, we carried out a post-trial landmark analysis (i.e., with the follow-up time beginning after trial closure). The landmark multivariate Cox model showed similar findings: among patients with left bundle-branch block who were alive at the completion of the original trial, treatment with CRT-D was associated with a significant survival benefit during the post-trial follow-up period, as compared with ICD therapy alone (adjusted hazard ratio, 0.58 ; 95\% confidence interval $[\mathrm{CI}], 0.39$ to $0.87 ; \mathrm{P}=0.009$ ); however, no corresponding benefit was observed in patients without left bundle-branch block (adjusted hazard ratio, $1.31 ; 95 \% \mathrm{CI}, 0.77$ to $2.23 ; \mathrm{P}=0.32$; $\mathrm{P}=0.02$ for interaction of treatment with $\mathrm{QRS}$ morphologic findings).

\section{DISCUSSION}

The findings of the present study provide evidence that early intervention with CRT-D is associated with a significant long-term survival benefit in patients with mild heart failure who have left ventricular dysfunction and an ECG pattern showing left bundle-branch block. However, there were no beneficial effects on long-term outcomes in patients without left bundle-branch block.

Cardiac-resynchronization therapy (CRT) with or without a defibrillator is associated with reverse remodeling and has been shown to reduce heart-failure symptoms and rates of hospitalization and death among patients with NYHA class III or IV heart failure. ${ }^{4-7}$ MADIT-CRT and the Resynchronization Reverses Remodeling in Systolic Left Ventricular Dysfunction (REVERSE) trial have shown that the echocardiographic and clinical benefit of CRT can be extended to the prevention of heart-failure progression in asymptomatic and mildly symptomatic patients (those in NYHA class I or II) with ischemic and nonischemic cardiomyopathy. ${ }^{1,8}$ In these trials, the mean follow-up time was only 2 to 3 years, raising the question of whether the early benefits of CRT in this population are sustained during long-term follow-up.

The Resynchronization-Defibrillation for Ambulatory Heart Failure Trial (RAFT) showed a reduction in the rate of death from any cause among patients with mild-to-moderate heart failure who were treated with CRT-D.9 This study, in contrast to ours, also enrolled patients with symptoms of more advanced heart failure (NYHA 


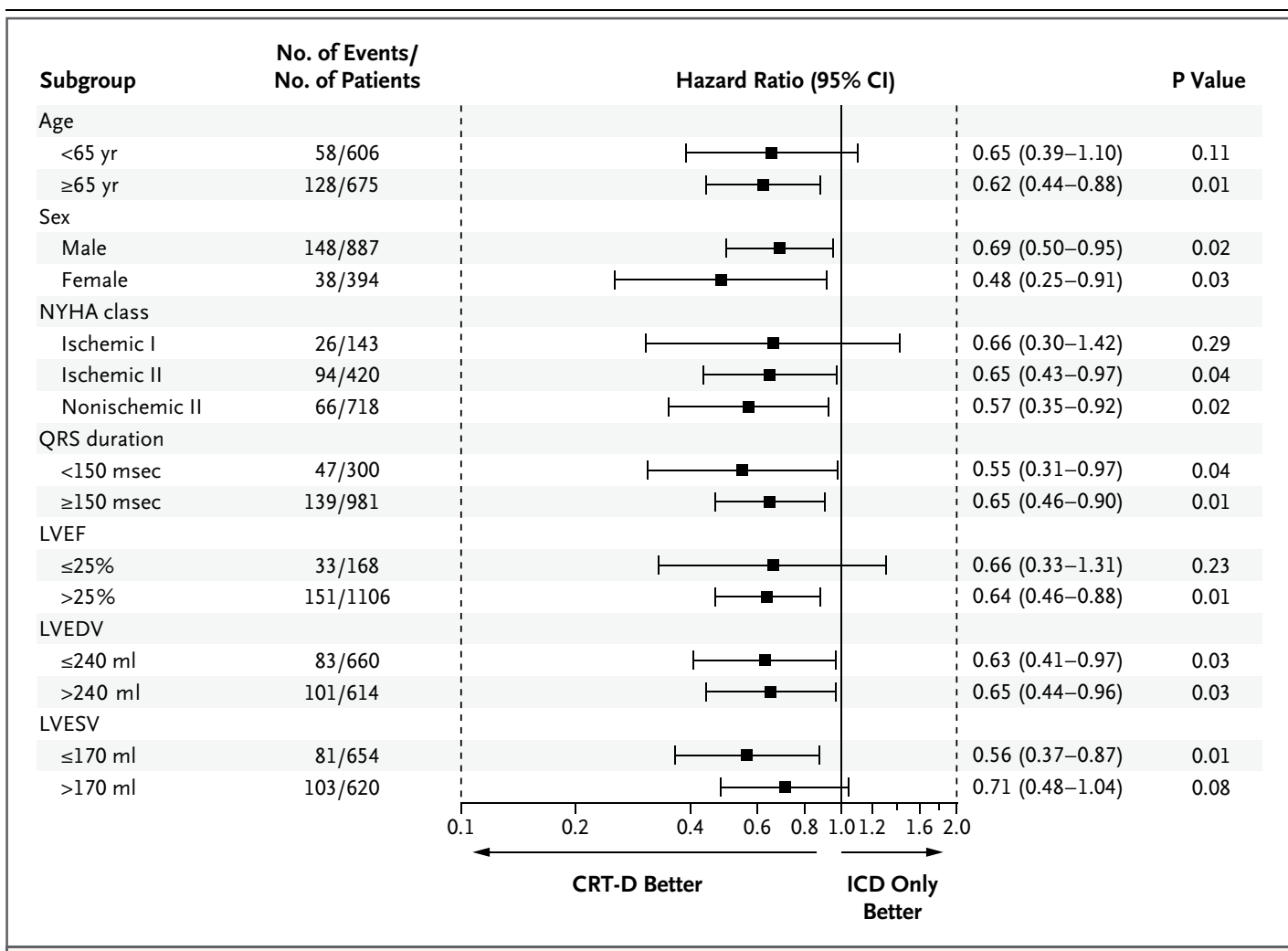

Figure 3. Risk of Death from Any Cause, According to Treatment Group, in Subgroups of Patients with Left BundleBranch Block.

Findings are based on univariate Cox proportional-hazards regression models and an interaction-term analysis. LVEDV denotes left ventricular end-diastolic volume, LVEF left ventricular ejection fraction, LVESV left ventricular end-systolic volume, and NYHA New York Heart Association.

class III). Thus, the findings of our study extend the RAFT data to patients with only mild heartfailure symptoms (NYHA class I or II) before device implantation.

Data from the in-trial phase of MADIT-CRT showed that treatment with CRT-D was associated with a pronounced reduction in the risk of both first and subsequent heart-failure events among patients with left bundle-branch block. There was no detectable effect regarding either of the two end points among patients without left bundle-branch block. ${ }^{2,10}$ The findings from the present study indicate that this treatment interaction was sustained during long-term follow-up. The long-term survival benefit of CRT-D in patients with left bundle-branch block was consistent in each subgroup analyzed, regardless of sex, QRS duration, and ischemic versus nonischemic cause of cardiomyopathy.

The mechanism underlying the differential effect of CRT-D according to QRS morphologic finding in MADIT-CRT is not clear. We previ- ously found that the patients in MADIT-CRT who had an ECG pattern that did not show left bundle-branch block had a significantly lower rate of echocardiographic response to CRT-D and a significant increase in the risk of ventricular tachyarrhythmic events after implantation of a CRT-D device, as compared with patients who had a pattern showing left bundle-branch block. ${ }^{11}$ These findings may be due to the fact that the spread of electrical activation in the left ventricular wall in patients without left bundlebranch block is more heterogeneous than in those with left bundle-branch block, ${ }^{12,13}$ possibly leading to a pacing-induced discrepancy of the wave front of the electrical activation, which has been shown to be associated with a poor prognosis. ${ }^{14}$ In the present study, the lack of a benefit of CRT-D in patients without left bundlebranch block was consistent, regardless of the QRS duration or presence or absence of right bundlebranch block or an intraventricular conduction delay. Thus, at present, our data do not support 
early intervention with CRT-D in any subset of this population.

Our findings are limited by a possible selection bias for the patients who were enrolled in phase 2 of the extended follow-up. It should be noted, however, that nearly all the patients who were lost to follow-up after phase 1 were from the U.S. medical centers that declined to participate in the phase 2 registry, suggesting that the possibility of individual patient bias is limited. It should also be noted that all the analyses in the present study were carried out on an intention-to-treat basis, thereby minimizing the possibility of a survival bias related to crossovers that occurring during or after the in-trial period.

Data from the present study clearly indicate that CRT-D was not beneficial during long-term follow-up in patients without left bundle-branch block who were enrolled in MADIT-CRT. However, the finding regarding a possible harmful effect of CRT-D in this population should be interpreted with caution, since it was obtained only after multivariate adjustment and is therefore sensitive to covariate selection.

In conclusion, our data from the MADIT-CRT long-term follow-up study provide evidence that treatment with CRT-D is associated with a significant long-term survival benefit in patients with mild heart failure who have a left ventricular ejection fraction of $30 \%$ or less and left bundle-branch block. However, we did not observe a clinical benefit in patients who had mild heart failure without left bundle-branch block.

Supported by unrestricted research grants from Boston Scientific to the University of Rochester School of Medicine and Dentistry and to the Israeli Association for Cardiovascular Trials.

Disclosure forms provided by the authors are available with the full text of this article at NEJM.org.

APPENDIX

From the Heart Research Follow-up Program, Division of Cardiology, Department of Medicine at the University of Rochester Medical Center, Rochester, NY (I.G., V.K., H.U.K., M.W.B., S.M., B.P., W.Z., A.J.M.); the Israeli Association for Cardiovascular Trials (I.G., A.D. R.K.), Leviev Heart Center, Sheba Medical Center (I.G., R.K.), Tel Hashomer, and Sackler School of Medicine, Tel Aviv University (I.G., S.V.) and Tel Aviv Sourasky Medical Center (S.V.), Tel Aviv - all in Israel; Cedars-Sinai Heart Institute and Hospital of the Good Samaritan, Los Angeles (D.S.C.); Department of Medicine, Duke University Medical Center, Durham, NC (J.P.D.); New England Cardiac Arrhythmia Center, Tufts Medical Center (N.A.M.E.), and Cardiovascular Division, Brigham and Women's Hospital and Harvard Medical School (M.A.P., S.D.S.) - all in Boston; Cardiology Unit, University of California at San Francisco, San Francisco (E.F.); Cardiology Unit, St. Luke's-Roosevelt Hospital, New York (H.G.); Cardiology Department, Institute for Clinical and Experimental Medicine, Prague, Czech Republic (J.K.); Kerckhoff Klinik, Bad Nauheim (M.K.), and Department of Medicine-Cardiology, University of Bonn, Bonn (A.G.) - both in Germany; Heart Center, Semmelweis University, Budapest, Hungary (B.M.); Hospital General de Valencia, Valencia, Spain (A.Q.); and the Cardiovascular Institute, Loyola University Medical Center, Chicago (D.W.).

\section{REFERENCES}

1. Moss AJ, Hall WJ, Cannom DS, et al. Cardiac-resynchronization therapy for the prevention of heart-failure events. N Engl J Med 2009;361:1329-38.

2. Zareba W, Klein H, Cygankiewicz I, et al. Effectiveness of cardiac resynchronization therapy by QRS morphology in the Multicenter Automatic Defibrillator Implantation Trial-Cardiac Resynchronization Therapy (MADIT-CRT). Circulation 2011;123:1061-72.

3. Moss AJ, Brown MW, Cannom DS, et al. Multicenter Automatic Defibrillator Implantation Trial-Cardiac Resynchronization Therapy (MADIT-CRT): design and clinical protocol. Ann Noninvasive Electrocardiol 2005;10:34-43.

4. Bristow MR, Saxon LA, Boehmer J, et al. Cardiac-resynchronization therapy with or without an implantable defibrillator in advanced chronic heart failure. N Engl J Med 2004;350:2140-50.

5. Cleland JG, Daubert JC, Erdmann E, et al. The effect of cardiac resynchronization on morbidity and mortality in heart failure. N Engl J Med 2005;352: 1539-49.

6. Cleland JG, Daubert JC, Erdmann E, et al. Longer-term effects of cardiac resynchronization therapy on mortality in heart failure [the CArdiac REsynchronization-Heart Failure (CARE-HF) trial extension phase]. Eur Heart J 2006;27:1928-32. 7. Bradley DJ, Bradley EA, Baughman $\mathrm{KL}$, et al. Cardiac resynchronization and death from progressive heart failure: a meta-analysis of randomized controlled trials. JAMA 2003;289:730-40.

8. Linde C, Abraham WT, Gold MR, St John Sutton M, Ghio S, Daubert C. Randomized trial of cardiac resynchronization in mildly symptomatic heart failure patients and in asymptomatic patients with left ventricular dysfunction and previous heart failure symptoms. J Am Coll Cardiol 2008;52:1834-43.

9. Tang AS, Wells GA, Talajic M, et al. Cardiac-resynchronization therapy for mild-to-moderate heart failure. $\mathrm{N}$ Engl J Med 2010;363:2385-95.

10. Goldenberg I, Hall WJ, Beck CA, et al. Reduction of the risk of recurring heart failure events with cardiac resynchronization therapy: MADIT-CRT (Multicenter Automatic Defibrillator Implantation Trial with Cardiac Resynchronization
Therapy). J Am Coll Cardiol 2011;58:72937. 11. Ouellet G, Huang DT, Moss AJ, et al. Effect of cardiac resynchronization therapy on the risk of first and recurrent ventricular tachyarrhythmic events in MADIT-CRT. J Am Coll Cardiol 2012;60: 1809-16.

12. Auricchio A, Fantoni C, Regoli F, et al. Characterization of left ventricular activation in patients with heart failure and left bundle-branch block. Circulation 2004; 109:1133-9.

13. Fantoni C, Kawabata M, Massaro R, et al. Right and left ventricular activation sequence in patients with heart failure and right bundle branch block: a detailed analysis using three-dimensional nonfluoroscopic electroanatomic mapping system. J Cardiovasc Electrophysiol 2005; 16:112-9.

14. Ypenburg C, van Bommel RJ, Delgado $\mathrm{V}$, et al. Optimal left ventricular lead position predicts reverse remodeling and survival after cardiac resynchronization therapy. J Am Coll Cardiol 2008;52: 1402-9.

Copyright (C) 2014 Massachusetts Medical Society. 